Journal for ImmunoTherapy of Cancer

\title{
VCN-01 disrupts pancreatic cancer stroma and exerts antitumor effects
}

\begin{abstract}
Miriam Bazan-Peregrino (D) , ${ }^{1}$ Rocio Garcia-Carbonero, ${ }^{2}$ Berta Laquente, ${ }^{3}$ Rafael Álvarez, ${ }^{4}$ Ana Mato-Berciano, ${ }^{1}$ Marta Gimenez-Alejandre, ${ }^{1}$ Sara Morgado, ${ }^{1}$ Alba Rodríguez-García, ${ }^{5,6}$ Maria V Maliandi, ${ }^{1} \mathrm{M}$ Carmen Riesco, ${ }^{2}$ Rafael Moreno, ${ }^{5}$ Mireia M Ginestà, ${ }^{7}$ Mercedes Perez-Carreras, ${ }^{8}$ Joan B Gornals, ${ }^{9}$ Susana Prados, ${ }^{4}$ Sofía Perea, ${ }^{4}$ Gabriel Capella, ${ }^{7}$ Ramon Alemany, ${ }^{5}$ Ramon Salazar, ${ }^{3}$ Emma Blasi, ${ }^{1}$ Carmen Blasco, ${ }^{1}$ Manel Cascallo, ${ }^{1}$ Manuel Hidalgo (D) 4,10
\end{abstract}

To cite: Bazan-Peregrino M, Garcia-Carbonero R, Laquente B, et al. VCN-01 disrupts pancreatic cancer stroma and exerts antitumor effects. Journal for ImmunoTherapy of Cancer 2021;9:e003254. doi:10.1136/ jitc-2021-003254

- Additional supplemental material is published online only. To view, please visit the journal online (http://dx.doi.org/10. 1136/jitc-2021-003254).

Accepted 11 September 2021

Check for updates

(c) Author(s) (or their employer(s)) 2021. Re-use permitted under CC BY-NC. No commercial re-use. See rights and permissions. Published by BMJ.

For numbered affiliations see end of article.

\section{Correspondence to} Dr Miriam Bazan-Peregrino; mbazan@vcnbiosciences.com

Dr Manuel Hidalgo; mah4006@med.cornell.edu

\section{ABSTRACT}

Background Pancreatic ductal adenocarcinoma (PDAC) is characterized by dense desmoplastic stroma that limits the delivery of anticancer agents. VCN-01 is an oncolytic adenovirus designed to replicate in cancer cells with a dysfunctional RB1 pathway and express hyaluronidase. Here, we evaluated the mechanism of action of VCN-01 in preclinical models and in patients with pancreatic cancer. Methods VCN-01 replication and antitumor efficacy were evaluated alone and in combination with standard chemotherapy in immunodeficient and immunocompetent preclinical models using intravenous or intratumoral administration. Hyaluronidase activity was evaluated by histochemical staining and by measuring drug delivery into tumors. In a proof-of-concept clinical trial, VCN-01 was administered intratumorally to patients with PDAC at doses up to $1 \times 10^{11}$ viral particles in combination with chemotherapy. Hyaluronidase expression was measured in serum by an ELISA and its activity within tumors by endoscopic ultrasound elastography.

Results VCN-01 replicated in PDAC models and exerted antitumor effects which were improved when combined with chemotherapy. Hyaluronidase expression by VCN01 degraded tumor stroma and facilitated delivery of a variety of therapeutic agents such as chemotherapy and therapeutic antibodies. Clinically, treatment was generally well-tolerated and resulted in disease stabilization of injected lesions. VCN-01 was detected in blood as secondary peaks and in post-treatment tumor biopsies, indicating virus replication. Patients had increasing levels of hyaluronidase in sera over time and decreased tumor stiffness, suggesting stromal disruption.

Conclusions VCN-01 is an oncolytic adenovirus with direct antitumor effects and stromal disruption capabilities, representing a new therapeutic agent for cancers with dense stroma.

Trial registration number EudraCT number: 2012-005556-42 and NCT02045589.

\section{INTRODUCTION}

Pancreatic ductal adenocarcinoma (PDAC) is one of the most fatal cancers. Despite significant research efforts, minimal progress has been achieved to date. The 5- year overall survival rate is still $<10 \%$ and has not substantially improved over the last 30 years. ${ }^{12}$ One of the hallmarks of PDAC is a dense stroma that penetrates and surrounds the neoplasm far exceeding what is found in other tumor types. ${ }^{3-6}$ This excess connective tissue, also known as 'tumor desmoplasia', constitutes approximately $80 \%$ of the tumor mass and contains fibroblasts, immune cells, and endothelial cells, embedded within a dense extracellular matrix, rich in collagen, fibronectin, periostin, and hyaluronic acid (HA). HA is a large linear glycosaminoglycan that is critical for the architecture, integrity, and malleability of tissues. More importantly, HA plays a major role in cancer-high HA levels correlate with invasive and metastatic cancer and poor prognosis. ${ }^{7-10}$

As noted, PDAC remains a significant unmet medical need. Surgery is the only treatment that offers the prospect of long termsurvival; however, the 5-year survival for the limited number of patients in whom resection is possible remains low $(20 \%-30 \%) .^{11}$ Patients with advanced disease are managed with chemotherapy. In recent years, the combination of gemcitabine with albuminbound paclitaxel, and the combination of folinic acid, 5-fluorouracil, irinotecan and oxaliplatin (FOLFIRINOX) have emerged as the standard of care. However, the results are still very poor and new therapeutic interventions are badly needed.

One of the major limiting factors for improving treatment efficacy in PDAC is the tumor stroma acting as a barrier for drug delivery. ${ }^{12} 13$ Studies have shown that HA expression plays a significant role in resistance to chemotherapy, and its degradation by pegylated hyaluronidase increases chemotherapy delivery and antitumor effects in preclinical models of PDAC, as well as in patients with high levels of HA. ${ }^{14-17}$ 
Consequently, targeting the components of the stromal compartment is a relevant approach for treating human tumors and is the rationale behind hyaluronidase expression in VCN-01.

VCN-01 is a genetically modified oncolytic type 5 adenovirus that selectively replicates within tumor cells that have deregulation of the retinoblastoma protein $(\mathrm{pRB})$ pathway, but not in normal cells. The virus has an integrinbinding motif RGDK inserted to replace the heparin sulfate glycosaminoglycan putative-binding site KKTK of the adenovirus 5 fiber, to increase tumor targeting and decrease hepatocyte tropism. It also expresses the human sperm hyaluronidase (PH20) that degrades the extracellular matrix. ${ }^{18}$ Taking into account the naturally lytic replication cycle of adenoviruses, together with the genetic modifications inserted in VCN-01, the biological activity of the VCN-01 oncolytic virus is based on the selective killing of tumor cells by its replication effects, which results in self-amplification of the initial inoculum that is released after cell lysis and spreads through the tumor mass; in addition, expression of a secreted soluble hyaluronidase, which partially degrades the extracellular matrix of the tumor, facilitates diffusion of the virus progeny ${ }^{19}$ and increases accessibility for other drug treatments. Here, we explored the activity and mechanism of action of VCN-01 in preclinical models of PDAC as well as in patients with the disease.

\section{MATERIALS AND METHODS \\ Preclinical studies}

\section{Oncolytic adenoviruses}

VCN-01 and ICOVIR15K have been previously described. ${ }^{18}$ All viruses were propagated in A549 cells and purified on a $\mathrm{CsCl}$ gradient according to standard techniques.

\section{In vivo efficacy studies}

Subcutaneous NP-9 or NP-18 tumors were established by injection of $7.5 \times 10^{6}$ or $5 \times 10^{6}$ cells, respectively, into the two flanks of a 6 -week-old male athymic nude mice (Harlan-Envigo). TP-11, a moderately differentiated pancreatic human tumor, ${ }^{20}$ has been perpetuated in nude mice by orthotopic implantation. Subcutaneous HP-1 tumors were established by injecting $5 \times 10^{6}$ cells into the flanks of a 6-week-old female Syrian Hamsters (Janvier). Once tumors reached $90 \mathrm{~mm}^{3}$ for NP-9, $150 \mathrm{~mm}^{3}$ for NP-18 and HP-1, $0.5 \mathrm{~cm}$ diameter for TP-11, animals were randomized and treated as described in the figure legend (figure 1). TRAMP-C2 tumors were established by the injection of $1 \times 10^{7}$ cells into both flanks of a 7-week-old male C57BL/6 (Envigo-Harlan). Once tumors reached a volume of $50 \mathrm{~mm}^{3}$ animals were randomized and treated as described in the figure legend (figure 2C). Anti-PD-L1 antibody was purchased from GoInVivo purified antimouse CD274, B7-H1, Ref: 124329, Biolegend. Tumor volume, body weight, and clinical signs were monitored at least twice per week. Tumor volume was defined by the equation $\mathrm{V}\left(\mathrm{mm}^{3}\right)=\pi / 6 \mathrm{xW}^{2} \mathrm{xL}$, where $\mathrm{W}$ and $\mathrm{L}$ are the width and length of the tumor, respectively.

To determine the intratumoral levels of gemcitabine, animals with orthotopic TP-11 tumors were treated with $50 \mathrm{mg} / \mathrm{kg}$ of gemcitabine (intraperitoneal, IP) 1 hour before sacrifice. Gemcitabine was extracted from 20 to $40 \mathrm{mg}$ of frozen tissue in acetonitrile $50 \%$ by homogenization $(0.05 \mathrm{mg}$ of tissue per $\mathrm{mL}$ of acetonitrile $50 \%)$. A $50 \mu \mathrm{L}$ of each homogenized sample was mixed with $200 \mu \mathrm{L}$ of internal pattern solution of labeled gemcitabine $(50 \mathrm{ng} / \mathrm{mL}$ of $13 \mathrm{C}, 15 \mathrm{~N} 2$-gemcitabine hydrochloride; Ref: C888 ALSA CHIM). Samples were centrifuged for $25 \mathrm{~min}$ at $20000 \mathrm{~g}$ and supernatants were evaporated in the SpeedVac. Samples were then reconstituted with $100 \mu \mathrm{L}$ of water and gemcitabine content was determined by high-performance liquid chromatography (HPLC) using a standard curve of gemcitabine (gemcitabine hydrochloride $\geq 98 \%$ (HPLC) G6423, SIGMA) diluted into an internal pattern of labeled gemcitabine.

\section{Pharmacokinetics and viral replication}

VCN-01 viral particles were measured in blood, tumors and liver over time. DNA was extracted from $\sim 25 \mathrm{mg}$ of frozen tissue using the QIAamp DNA Mini Kit (Qiagen). A $20 \mu \mathrm{L}$ of blood was placed in phosphate-buffered saline (PBS) $(180 \mu \mathrm{L})$ and frozen. DNA was extracted using QIAamp DNA Mini Kit (Qiagen). Quantification of viral genomes was performed by qPCR using a LightCycler 480 II and SYBR green (Roche) with the following primers: Forward 5' - CTT CGA TGA TGC CGC AGT G-3'; Reverse 5'-ATG AAC CGC AGC GTC AAA CG- 3'.

\section{E1A and HA histochemical staining}

Paraffin-embedded blocks were cut into $4 \mu \mathrm{m}$ thick sections. Serial sections were employed for E1A and HA staining. For E1A staining, anti-E1A antibody was diluted 1/200 in PBS (sc-25 mouse monoclonal, Santa Cruz Biotechnology) and incubated overnight at $4^{\circ} \mathrm{C}$. Slides were then washed in PBS $+0.2 \%$ tritonX-100 and treated with secondary antibody (Dako EnVision+System HRP Labeled Polymer Anti-mouse, Dako Laboratories, Glostrup, Denmark) for $30 \mathrm{~min}$ at RT. For HA staining, slides were incubated with $5 \mu \mathrm{g} / \mathrm{mL}$ of a bionylated HA binding protein (Seikagaku, Japan) overnight at $4^{\circ} \mathrm{C}$. Slides were then washed in PBS and treated with avidin-biotin-peroxidase kit (ABC KIT PK-4000, Vectastain. Burlingame, California, USA). For either E1A or HA, sections were developed with DAB (Dako Laboratories, Glostrup, Denmark), counterstained with hematoxylin, dehydrated and mounted.

In vivo imaging of tumor extravasation of labelled anti-PD-L1 antibody

To evaluate the extravasation of antibodies within treated tumors, we administered animals labeled antiprogrammed death-ligand 1 ( $\alpha$-PD-L1) antibody. Antibody $\alpha$-PD-L1 antibody (InVivoMAB anti-human PD-L1, B7-H1, Clon 29E.2A3 Ref: BE0285, BioXCell) was labeled with the fluorochrome VivoTag 800 (Ref: NEV11108, 
A)

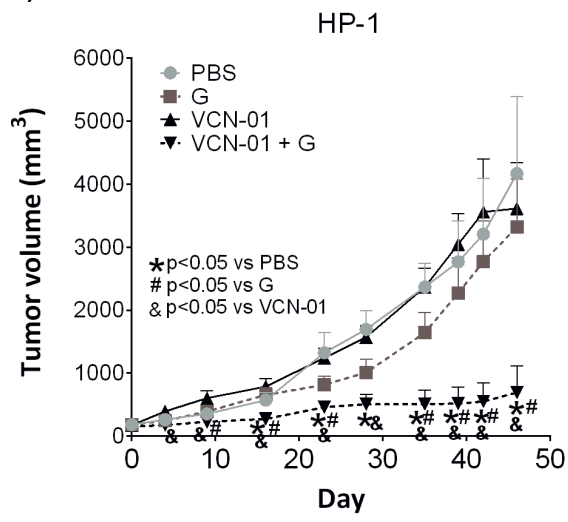

C)

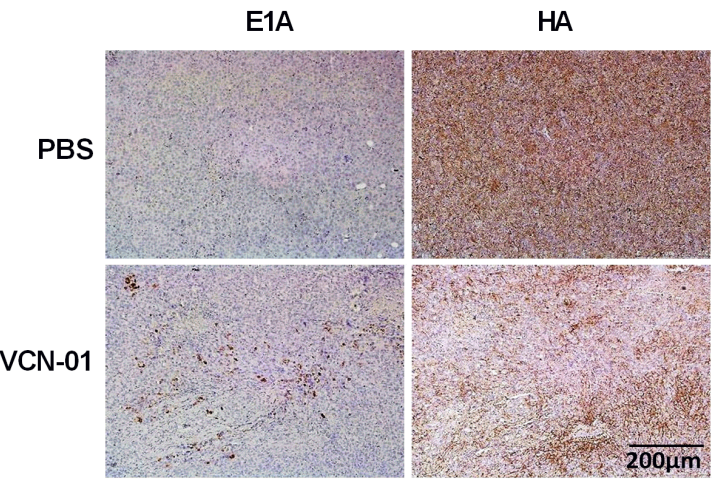

E)

NP-18 VCN-01 + GA

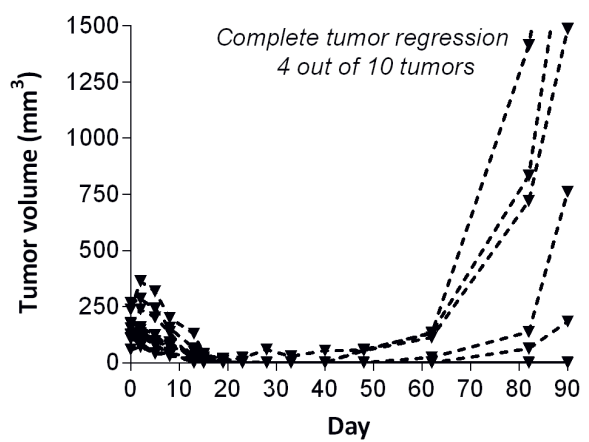

B)

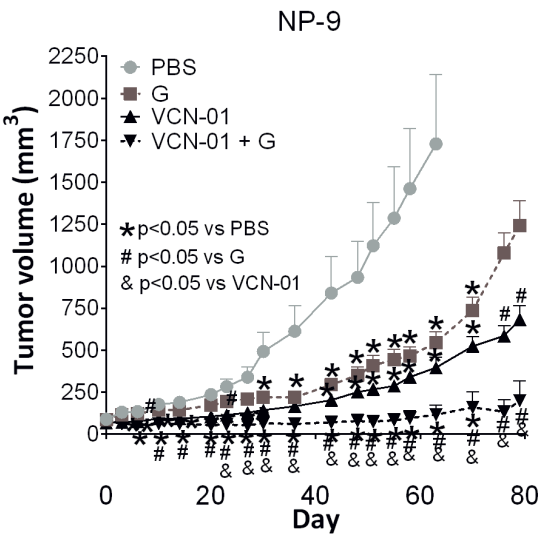

D)

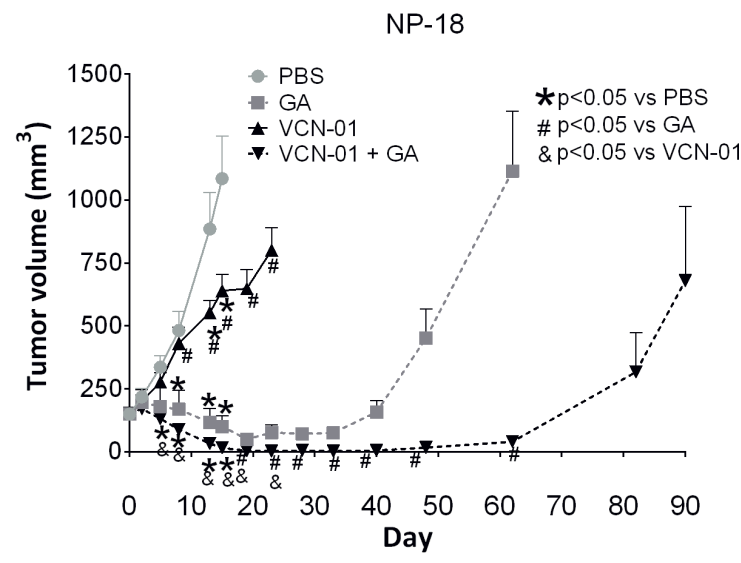

F)

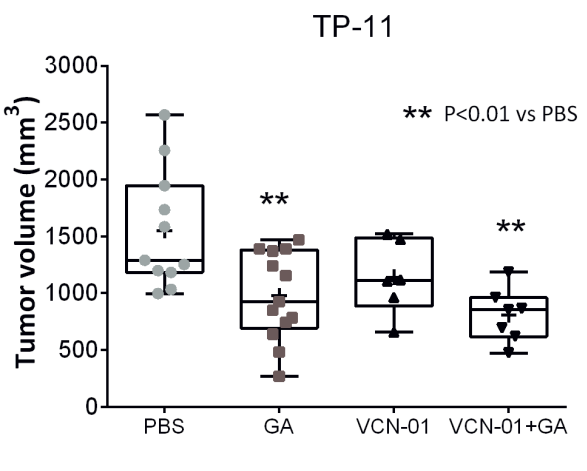

Figure 1 VCN-01 sensitizes tumors to chemotherapy leading to enhanced antitumor activity. (A) Antitumor activity against subcutaneous HP-1 Syrian hamster pancreatic tumors, implanted in immunocompetent hamsters, was evaluated after three intratumoral administrations of $2.5 \times 10^{10} \mathrm{vp}$ of $\mathrm{VCN}-01$ per tumor alone (days 0,9 and 18) or in combination with intraperitoneal (IP) $\mathrm{G}$ for seven doses (50 mg/kg, days 0, 3, 6, 9, 12, 15 and 18). $\mathrm{n}=8-12$ tumors/group. (B) Antitumor activity against subcutaneous human NP-9 pancreatic tumors, implanted in immunodeficient mice, was evaluated after single intravenous administration of $4 \times 10^{10}$ viral particles (VP) of VCN-01 alone (day 0) or in combination with IP gemcitabine (G) $(50 \mathrm{mg} / \mathrm{kg}$, days $0,3,6$ and 9). $n=7-13$ tumors/group. (C) Viral E1A expression and hyaluronic acid (HA) degradation indicative of viral replication as determined by immunohistochemistry on serial tumor sections of VCN-01 treated tumors of (B) on day 79. (D) Antitumor activity against subcutaneous human pancreatic NP-18 tumors, implanted in immunodeficient mice, was evaluated after single intravenous administration of $\mathrm{VCN}-01\left(4 \times 10^{10} \mathrm{vp} /\right.$ animal, day 0$)$ with and without IP G with nab-paclitaxel plus gemcitabine (GA) (G 50 mg/kg and a $41.6 \mathrm{mg} / \mathrm{kg}$, days 0,7 and 14$)$. $n=10-12$ tumors/group. (E) Tumor volumes for each tumor treated with VCN-01 +GA. For A, B, D, a parametric t-student test was used to determine statistical significance of the different treatments at each time point. (F) Antitumor activity against the orthotopic human TP-11 pancreatic model, implanted in immunodeficient

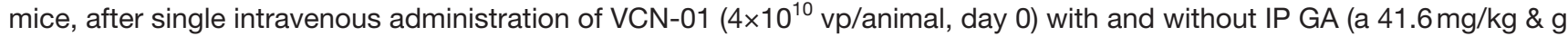
$50 \mathrm{mg} / \mathrm{kg}$, days 0,7 and 14) and measured tumor volume at the experiment end (day 28$) . n=6-13$ tumors/group. Mean $\pm S E M$ is plotted (black lines). One-way ANOVA (Tukey's multiple comparisons test) was used to determine statistical significance of the different treatments. ANOVA, analysis of variance; GA, gemcitabine with albumin. 
A)

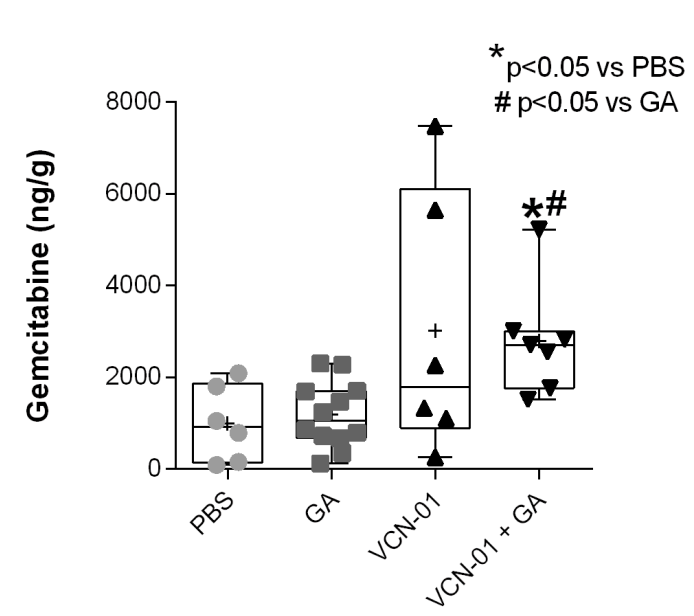

B)

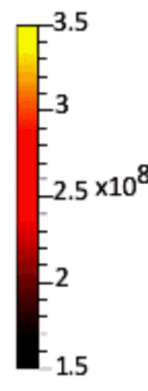

\section{PBS ICOVIR15K VCN-01}

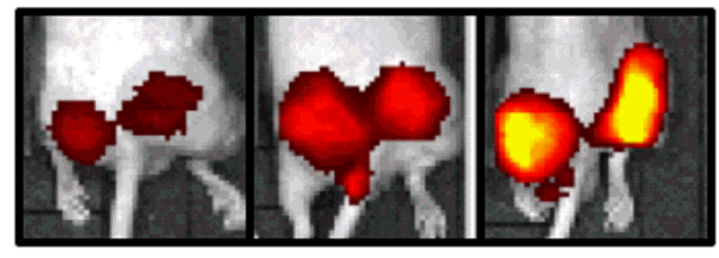

Radiant Efficiency

$\left[\mathrm{p} / \mathrm{s} / \mathrm{cm}^{2} / \mathrm{sr}\right] /\left[\mu \mathrm{W} / \mathrm{cm}^{2}\right]$

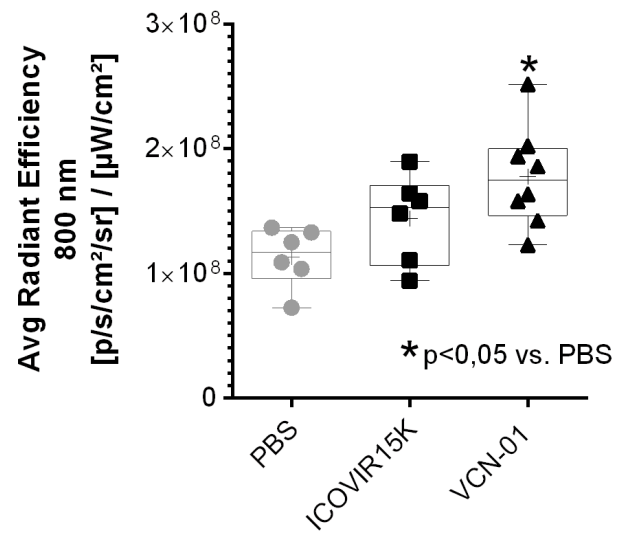

C)

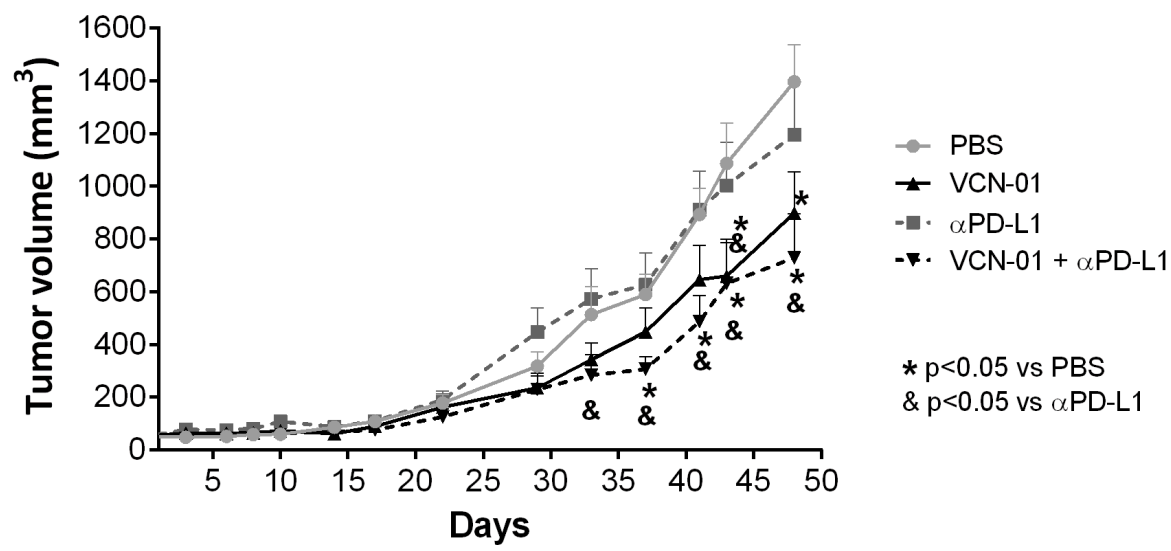

Figure 2 VCN-01 enhances extravasation of chemotherapy and monoclonal antibodies into tumors. (A) Single intravenous administration of VCN-01 alone or in combination with chemotherapy significantly increases tumor-uptake of gemcitabine (G) in an orthotopic pancreatic model, TP-11. Animals from the different experimental groups described in figure $1 \mathrm{~F}$ were administered G IP at a dose of $50 \mathrm{mg} / \mathrm{kg} 1$ hour before sacrifice. The intratumoral content of $\mathrm{G}$ in each tumor was measured by high-performance liquid chromatography (HPLC). Box and whiskers plots (mean is shown as + ). $n=6-13$ tumors/group. Oneway ANOVA (Tukey's multiple comparisons test) was employed to determine statistical significance of the different treatments. (B) fluorescence in vivo imaging after intravenous administration of labeled anti-PD-L1 antibody $(10 \mathrm{mg} / \mathrm{kg})$. VCN-01 or the hyaluronidase negative virus ICOVIR15K was administered intravenous at a dose of $4 \times 10^{10} \mathrm{vp} /$ animal to NP-18 tumor-bearing immunodeficient mice on day 0. Labeled anti-PD-L1 (Vivotag 800) was administered intravenous at a dose of $10 \mathrm{mg} / \mathrm{kg}$ on day 14 and imaging performed after 48 hours. Upper panel: representative images from fluorescence in tumors show enhanced extravasation of labeled anti-PD-L1 in VCN-01 treated tumors compared with the ICOVIR15K group. Lower panel: quantification of average radiant efficiency at $800 \mathrm{~nm}$ in tumor areas is represented in the box and whiskers plot (mean is shown as + ). $n=6-8$ tumors/group. One-way ANOVA (Tukey's multiple comparisons test) was employed to determine statistical significance of the different treatments. (C) tumor growth curves of the TRAMPC-2 cancer model treated with $1 \times 10^{10} \mathrm{vp} / \mathrm{tumor}$ of VCN-01 or phosphate-buffered saline administered intratumorally alone or in combination with five intraperitoneal doses of $200 \mu \mathrm{g}$ of antiPD-L1 antibody (days 0, 3, 6, 8 and 10). $n=8-9$ tumors/group. A two-way ANOVA, followed by a Tukey's multiple comparisons test, was employed to determine statistical significance of the different treatments for each day. ANOVA, analysis of variance; GA, gemcitabine with albumin. 
Perkin Elmer), following the manufactures' protocol $(0.3 \mathrm{mg}$ of fluorochrome per $3 \mathrm{mg}$ of antibody). Labeled anti-human PD-L1 was administered intravenous at $10 \mathrm{mg} / \mathrm{kg}$ intravenous on day 15 and fluorescence signal was quantified after 48 hours in tumors by IVIS ${ }^{\circledR}$ (Lumina $\mathrm{XR}$, Caliper LifeSciences) as average radiant efficiency at $800 \mathrm{~nm}\left(\left[\mathrm{p} / \mathrm{s} / \mathrm{cm}^{2} / \mathrm{sr}\right] /\left[\mu \mathrm{W} / \mathrm{cm}^{2}\right]\right)$.

\section{Clinical trial}

Study design

This was a multicenter, phase 1 dose escalation study of intratumorally (IT) VCN-01 in combination with standard doses/schedules of either gemcitabine or nab-paclitaxel plus gemcitabine. All patients gave written informed consent prior to study enrollment. Online supplemental figure 1 provides a schema of the phase I trial design. The primary objective was to determine the safety and tolerability of the combination and the recommended phase 2 dose. Secondary objectives included assessment of viral particles in paired tumor biopsies, measurement of tumor stiffness by ultrasound elastography and circulating levels of PH20.

\section{Eligibility criteria}

Key inclusion criteria included confirmed histologic diagnosis of unresectable PDAC amenable to endoscopic ultrasound-guided (EUS) injection; adequate baseline organ function (hematologic, liver, renal and nutritional) and status of ECOG PS 0-1. Key exclusion criteria were previous treatment with live attenuated vaccines in the last 3 weeks; viral syndrome diagnosed during the 2 weeks before inclusion; chronic liver disease; and chronic immunosuppressive therapy.

\section{Treatment schema}

VCN-01 was administered IT at two different dose levels. At the first dose level of VCN-01, cotreatment with gemcitabine was the choice as it was the standard of care when the clinical trial started, but then standard practice changed to the combination with nab-paclitaxel plus gemcitabine and this was given with the second dose level. In dose level $1,1 \times 10^{10}$ vp of VCN-01 was administered on days 1 , 22 , and 43 in combination with gemcitabine at $1000 \mathrm{mg} /$ $\mathrm{m}^{2}$ intravenous on days $1,8,15,22,29,35$, and 43. Dose level two consisted of VCN-01 $1 \times 10^{11}$ vp on days 1,29 and 57 in combination with nab-paclitaxel plus gemcitabine $\left(125 / 1000 \mathrm{mg} / \mathrm{m}^{2}\right.$ intravenous) on days 1,8 and 15 and then every 4 weeks. After day 56, patients continued to receive standard chemotherapy until disease progression, unacceptable toxicity, consent withdrawal or investigator's decision.

\section{Assessments}

Patients were initially evaluated by a medical history and physical exam. Eligibility criteria including performance status, adequate liver, kidney and bone marrow function were assessed at baseline and periodically during study participation. Response to treatment was assessed by CT every 8 weeks and reported as per the RECIST V.1.1 criteria. Antitumor efficacy in injected tumor lesions was measured by computerized tomography (CT), Positron Emission Tomography (PET)-CT or eco-endoscopy (longest diameter) and graphed as the percentage change in tumor burden. Once a new lesion appeared and the patient progressed, the injected lesion was not monitored. Toxicity was monitored continuously and graded as per the CTCAE V.4.03 criteria. ARs were coded according to the Medical Dictionary for Regulatory Activities system.

The presence of VCN-01 in tumor samples was determined by fine-needle aspiration (FNA) biopsy of the tumor taken at baseline and at one time point posttreatment either on day 20, 21, or 28 and frozen until analysis. A $200 \mu \mathrm{L}$ of patient blood was also analyzed for the presence of VCN-01 only after the first viral injection on days $1,2,3,8,15,22,29$, and 36. DNA was extracted using QIAamp DNA Blood Mini Kit (Qiagen) and quantified with a Nanodrop 2000 (Thermo Scientific). Quantification of viral genomes was performed by qPCR using a LightCycler 480 II and SYBR green (Roche) with these primers: Forward: 5"- ACA TTG CCC AAG AAT AAA GAA TCG - 3". Reverse: 5"- TGG AAT CAG AAG GAA GGT GAA - 3. Viral DNA quantification in the tumor was expressed as viral particles per $\mu \mathrm{g}$ of DNA, whereas for blood it was expressed as viral particles per $\mathrm{ml}$ of blood.

PH20 serum levels were tested before and after treatment using ELISA (Human Hyaluronidase PH-20 (SPAM1) ELISA kit, Ref: CSB-EL022471HU, Cusabio). Human serum samples were diluted $1 / 2$ in sample diluent and analyzed in duplicate. Optical densities at $450 \mathrm{~nm}$ and $540 \mathrm{~nm}$ were measured on a microplate reader (Perkin Elmer, EnSpire). After subtracting the reading at $540 \mathrm{~nm}$ from the reading at $450 \mathrm{~nm}$ (to correct optical imperfections in the plate), PH20 concentration for each sample was calculated by the absorbance interpolation of the standard curve (Sigmoidal, 4PL, $\mathrm{X}$ is $\log ($ concentration $))$.

Tissue stiffness was measured by EUS elastography. ${ }^{21-23}$ The elasticity of representative areas of the tumor was analyzed and compared with the elasticity of surrounding areas of healthy reference tissue. Elastographic evaluation was interpreted using the strain ratio which is the result of the quotient: elasticity of reference areas/elasticity of tumor. When tumor elasticity increases (due to decreased stiffness), strain ratio decreases.

\section{Statistical analysis}

A parametric Student's t-test was used to determine the statistical significance of the different treatments at each time point for body weight and tumor volume over time. One-way analysis of variance (Tukey's multiple comparisons test) was used to evaluate multiple comparisons between groups in the following experiments: gemcitabine and antibody extravasation, antitumor activity at a single time point in TP-11, biochemistry, hematology parameters and PH20 serum levels. A Mann-Whitney $\mathrm{U}$ test was performed to analyze statistically significant 
differences between two groups in viral replication analysis.

\section{RESULTS}

\section{Preclinical efficacy studies of VCN-01 and chemotherapy}

We hypothesized that VCN-01 could sensitize tumor cells to chemotherapy. Thus, we evaluated the efficacy of VCN-01 in combination with gemcitabine or nab-paclitaxel plus gemcitabine in immunodeficient and immunocompetent preclinical models of PDAC (figure 1).

First, we evaluated the efficacy of the combination of VCN-01 plus gemcitabine in the immunocompetent Syrian hamster with HP-1 subcutaneous pancreatic tumors (figure 1A). We administered VCN-01 IT alone or in combination with IP gemcitabine. Neither gemcitabine nor VCN-01 alone induced antitumoral effect in this hamster tumor model. Limitation posed by the semipermissiveness of human adenovirus in the hamster model is likely to have a strong impact on the efficacy of VCN-01 alone. However, the combination of IP gemcitabine and IT VCN-01 resulted in a synergistic antitumoral effect which was significantly superior to control and either treatment alone. These results indicate that VCN-01 induces powerful chemosensitization to gemcitabine treatment in the semipermissive Syrian hamster model (figure 1A) and confirmed the observed in vitro synergistic effects of VCN-01 plus gemcitabine summarized in online supplemental table 1.

We also compared a single dose of intravenous VCN-01 plus four doses of IP gemcitabine to either treatment alone in the HA rich human pancreatic NP-9 tumor model grown subcutaneously in athymic mice (figure 1B). VCN-01 antitumor activity was superior to gemcitabine alone, and the combination of both treatments was significantly better than either single treatment alone for controlling tumor growth up to day 79 (figure 1B). VCN-01 treated tumors were positive for the key viral replication gene, E1A, and showed decreased HA content at the study end confirming that the transgene hyaluronidase PH20 was functionally expressed (figure 1C).

We next tested intravenous VCN-01 in combination with nab-paclitaxel plus gemcitabine in a subcutaneous human pancreatic tumor model (NP-18). VCN-01 alone significantly decreased tumor growth compared with untreated tumors in the NP-18 model (figure 1D). Nabpaclitaxel plus gemcitabine reduced tumor volume in all treated tumors, leading to complete regression in $25 \%$ of $(3 / 12)$ tumors. However, all regressed tumors relapsed after stopping chemotherapy administration. The combination of VCN-01 plus nab-paclitaxel plus gemcitabine reduced tumor growth to a greater extent than both treatments administered separately, leading to complete regression in $80 \%(8 / 10)$ of tumors. In addition, $50 \%$ $(4 / 10)$ of these tumors continued with complete regression until the end of treatment (day 90) (figure 1E).

We next selected the TP-11 model because of its high HA content and architectural resemblance to human
PDAC when implanted orthotopically (online supplemental figure 2). In this model, all treated groups demonstrated reduced tumor volume compared with nontreated animals on day 28. There was a minor nonsignificant reduction in tumor volume due to VCN-01 $(27 \%)$, whereas a significant reduction was observed with nab-paclitaxel plus gemcitabine $(37 \%)$ and a further significant decrease was achieved with the combined treatment (VCN-01 and nab-paclitaxel plus gemcitabine) (48\%) (figure 1F). In a separate experiment, using a slightly longer evaluation timepoint (day 36), VCN-01 showed a significant tumor reduction (29\%) in the TP-11 tumor model (online supplemental figure 3).

Treatment with VCN-01 alone and in combination with chemotherapy was well tolerated (online supplemental figure 4).

\section{Preclinical evaluation of viral replication and drug extravasation}

Viral replication within the tumor is the basic mechanism of antitumor activity of oncolytic viruses. This process results in shedding of viral particles into the bloodstream that is reflected by a secondary or delayed peak of viremia.

After IT administration in mice with subcutaneous NP-18 tumors, VCN-01 leaked into the blood 2 min after viral administration, with similar viral blood levels up to $60 \mathrm{~min}$ and progressive clearance from 4 to 24 hour thereafter. VCN-01 was detected in blood again after 4 days and continued to be detected until the last day of analysis (day 29 ), suggesting ongoing viral replication in tumor tissues (figure 3A).

The pharmacokinetics of $\mathrm{VCN}-01$ after intravenous administration (in NP-18 tumor model) showed a biphasic pattern, with an initial rapid decrease in plasma concentration in less than $5 \mathrm{~min}$, and a sustained and constant decrease up to 24 hours. We observed a second peak of viremia at 48 hours, after which the virus progressively cleared from blood, being detected up to day 21 (figure 3A).

We next evaluated viral replication of VCN-01 in tumor tissues. As shown in figure 3C, intravenous administration of VCN-01 led to effective viral replication in orthotopic pancreatic tumors (TP-11), reaching a 3-log increase from day 2 to day 28. As expected, VCN-01 accumulated in the liver after intravenous injection. Contrary to what occurs in the tumor, VCN-01 did not replicate within the liver and was eliminated over time, demonstrating a 2-log decrease from day 2 to day 28 . The main limitation of this immunodeficient model when translating it to patients is the lack of functional T-cells which may help clearing the virus faster in both the tumor and the liver and initiate an antitumor immune response. In addition, after systemic administration, VCN-01 replicated in both subcutaneous human xenografts and orthotopic pancreatic tumors, regardless of chemotherapy cotreatment (online supplemental figure 5 ).

To elucidate the mechanism of action underlying the increased antitumor efficacy elicited by the combination 
A)

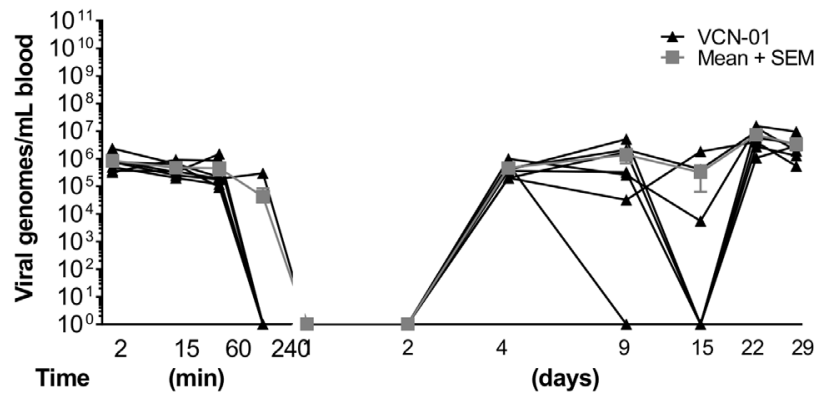

B)
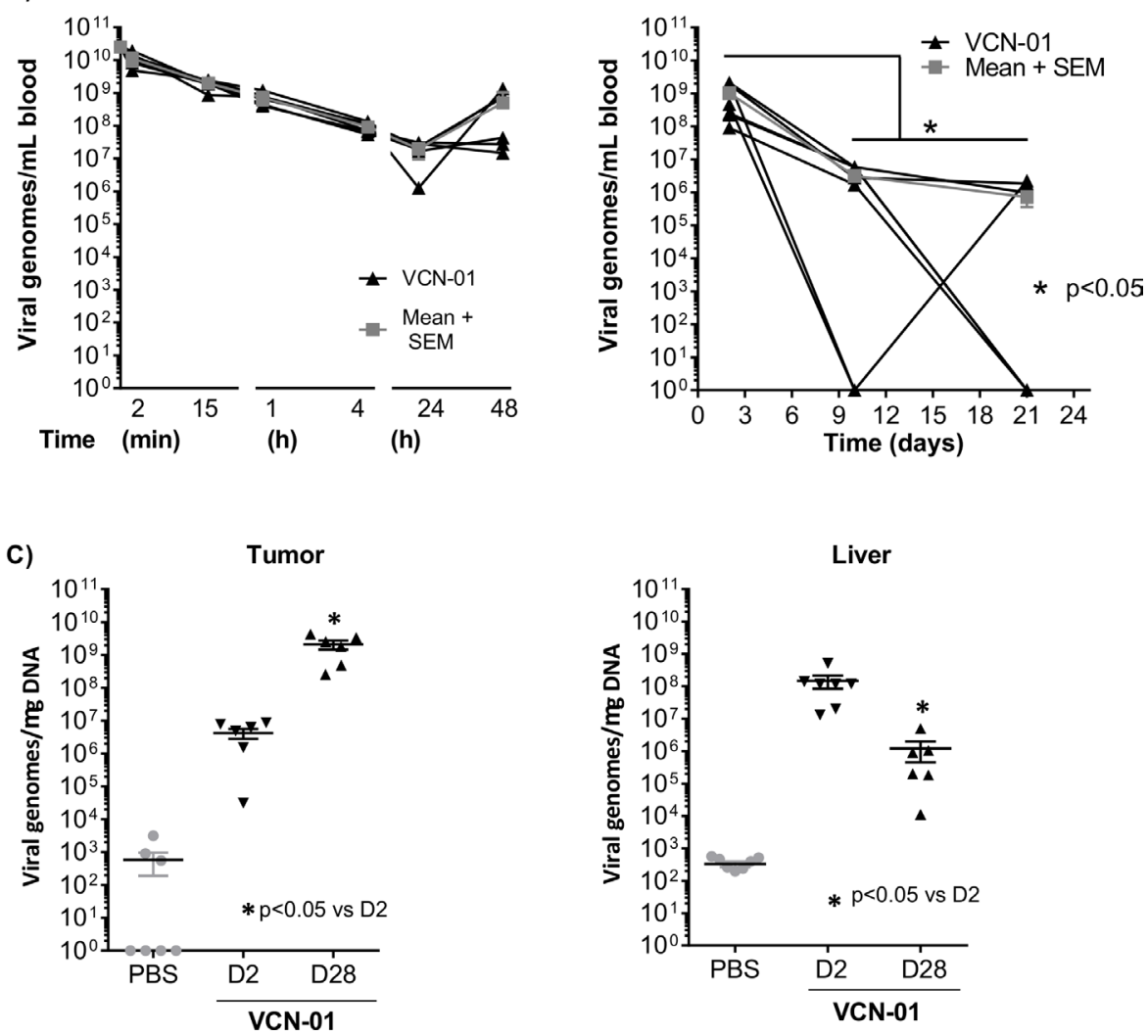

Figure 3 VCN-01 pharmacokinetics in mice and intratumoral viral replication. (A) Pharmacokinetics and peaks of replication of VCN-01 in blood after single intratumoral administration of $5 \times 10^{8} \mathrm{vp}$ in athymic mice with the human pancreatic tumor NP-18 implanted subcutaneous (SC). Blood samples $(20 \mu \mathrm{L})$ were obtained at the indicated time points postadministration and viral genomes were measured by qPCR. $n=6-7$ mice/time point. (B) Left panel: VCN-01 blood levels after administration of a single intravenous dose of $5 \times 10^{10} \mathrm{vp} / \mathrm{animal}$ to immunodeficient mice bearing the SC human pancreatic NP-18 tumor. Blood samples $(20 \mu \mathrm{L})$ were obtained at 2 and $15 \mathrm{~min}$ and at 1, 4, 24, and 48 hours. Post dose and genomes were measured by qPCR. Right panel: VCN-01 viral genomes in blood of immunodeficient mice with the SC NP-18 human pancreatic model after intravenous administration of $4 \times 10^{10} \mathrm{vp} /$ animal of $\mathrm{VCN}-01$ on day 0 . Blood samples $(20 \mu \mathrm{L})$ were collected on days 2,10 and 21 . Results are expressed as viral genomes per $\mathrm{mL}$ of total blood. $\mathrm{n}=6-7$ mice/time point. (C) Viral genomes in tumors and livers were analyzed at the indicated time points after one time intravenous administration of VCN- $01\left(4 \times 10^{10} \mathrm{vp} / \mathrm{animal}\right)$ to mice with orthotopically implanted TP-11 human pancreatic tumor model. Animals were sacrificed on day 2 and 28 and VCN-01 genome measured by qPCR. $n=6-8$ tumors or livers/group/day. A Mann-Whitney $U$ test was employed to analyze statistically significant differences $\left({ }^{*} \mathrm{p}<0.05\right.$ vs D2).

of VCN-01 and chemotherapy, we investigated the role of hyaluronidase PH20 encoded by VCN-01 as a facilitator of drug extravasation into the tumor. Animals were treated with nab-paclitaxel plus gemcitabine, VCN-01, or the combination thereof. After 28 days, all animals received a dose of IP gemcitabine prior to sacrifice to assess gemcitabine distribution to the tumor. As shown in figure 2A, pretreatment with
VCN-01 and nab-paclitaxel plus gemcitabine led to higher IT levels of gemcitabine on day 28 compared with nabpaclitaxel plus gemcitabine alone. We observed the same trend in tumors treated with VCN-01 alone, although this did not reach statistical significance due to high interanimal variability (figure 2A). These results support a role of hyaluronidase for better drug extravasation. 
To further test if hyaluronidase PH20 expression from VCN-01 increases the delivery of anticancer agents to tumor tissues, we compared the impact on IT levels of an anti-PD-L1 antibody by cotreatment with VCN-01 or ICOVIR15K, an identical oncolytic adenovirus that lacks hyaluronidase PH20 expression. We administered oncolytic adenoviruses as a single intravenous dose in athymic mice with the PD-L1 ${ }^{+}$NP-18 model. The antiPD-L1 antibody was labeled with the near-infrared fluorochrome Vivotag 800, and then injected in all groups on day 15 and imaged after 48 hours. We observed a minor non-significant increase in extravasation of PD-L1 antibody after treatment with the control (ICOVIR15K lacking hyaluronidase expression). However, VCN-01 significantly increased extravasation of the antibody into PD-L1 ${ }^{+}$NP-18 tumors compared with non-treated (PBS) tumors (figure 2B). This further corroborates the role of hyaluronidase in increasing delivery, not only of chemotherapy, but also of large molecules such as monoclonal antibodies. Accordingly, single IT administration of VCN-01 in combination with anti-PD-L1 antibody treatment resulted in significant decrease in tumor growth in immunocompetent mice with TRAMP-C2 tumors (figure 2C). Of note, VCN-01 can hardly replicate in this immunocompetent mouse model due to the limitation posed by the semi-permissiveness of human adenovirus.

\section{Clinical study}

The mechanisms of action of VCN-01 was subsequently evaluated in a phase 1 trial including eight patients where the safety and efficacy of IT VCN-01 in combination with gemcitabine (first two patients) or gemcitabine/nab-paclitaxel (subsequent six patients when SoC changed) was analyzed (online supplemental figure 1). Their demographic characteristics are depicted in online supplemental table 2. Six patients had metastatic disease and two had locally advanced disease. One received prior chemotherapy for pancreatic cancer. VCN-01 was administered to primary tumor lesions by EUS guided.

Treatment-related adverse events were dose-dependent and mainly consisted of asthenia (six patients), fever (four patients), and transaminase increases (three patients). Online supplemental table 3 summarizes safety data. There were three episodes of dose-limiting toxicities in three patients treated at the highest dose level tested $\left(1 \times 10^{11} \mathrm{vp} /\right.$ patient $)$. Two patients had a grade 3 increase in transaminases that spontaneously regressed. Another patient developed a pancreatic fistula with intraabdominal fluid collection, associated with a gastric necrotic ulcer and a severe upper GI hemorrhage that resulted in death. This event was considered treatment related and was associated with extensive necrosis and a completely lysed tumor mass at the injection site that could be potentially related to VCN-01 replication. Overall, the safety profile was consistent with previous reports for other oncolytic adenoviruses and other adenovirus-based products injected by EUS. ${ }^{24}$

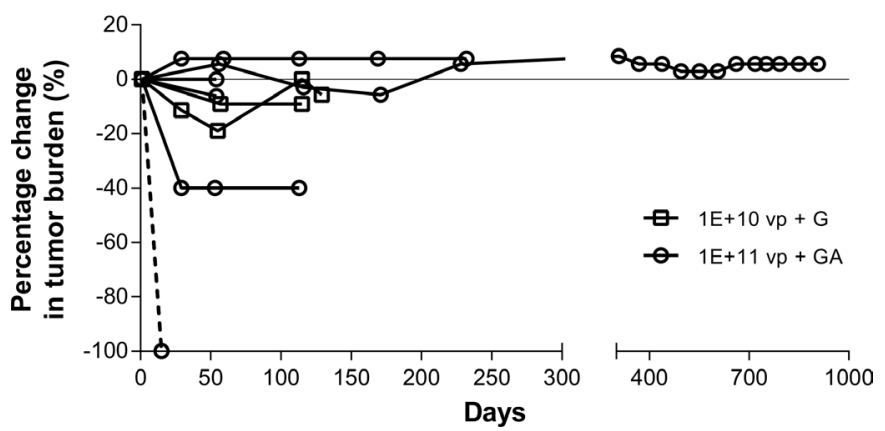

Figure 4 Antitumor activity of Intratumor Injections of VCN-01 in patients. Percentage variation in the size of tumor lesions injected with VCN-01 compared with baseline in patients treated with VCN-01 in combination with either gemcitabine (G) or nab-paclitaxel plus gemcitabine (GA). Ech blue line represents the monitored tumor of each patient (eight patients).

In all treated patients, the injected lesion remained stable or decreased in size. Of seven evaluable patients, five progressed at 4 months, one at 8 months and one at 31 months after treatment. All patients progressed because of appearance of new lesions or growth of distant, noninjected, metastatic lesions (figure 4). Furthermore, the patient who died on day 22 of massive intra-abdominal bleeding showed extensive tumor necrosis on CT scan on day 15 of the pre-existing $4.4 \mathrm{~cm}$ pancreatic tumor mass and was attributed to a rapid oncolytic effect of VCN-01 on the injected lesion.

\section{Mechanistic studies in patients}

We detected VCN-01 in blood samples after IT delivery on day 1 of treatment, most likely due to leakage into the blood system. Subsequently, we observed a decrease in VCN-01 genomes over time reaching a nadir on day 3, followed by a biphasic surge in viremia with a first peak of virus in blood observed on day 8 and a second peak on day 28 suggesting tumor viral replication (figure 5A). We obtained paired tumor biopsies before and after treatment in six patients. As expected, we did not observe VCN-01 at baseline, but detected it in all six patients between day 21 and 28 consistent with viral replication in the tumor cells (figure 5B).

To assess if the pre-existing anti-adenovirus Ad5 levels (NAbs) have an impact on efficacy or on biodistribution, the data was evaluated by Spearman's correlation. No correlations were observed between baseline anti-Ad5 Nabs levels and efficacy or biodistribution. This may be attributed to the route of administration that was intratumoral (online supplemental figure 6).

We next assessed production of PH20 by VCN-01 as quantification of PH20 serum levels offers a method to monitor VCN-01 replication. As shown in figure 6A, we observed an increase in serum levels of PH20 over time, reaching maximum levels on days 3 to 36 (but only day 36 was significant). As PH20 partially degrades the extracellular matrix, we hypothesized that VCN-01 could reduce tumor stiffness. To test this hypothesis, EUS-elastography 
A)

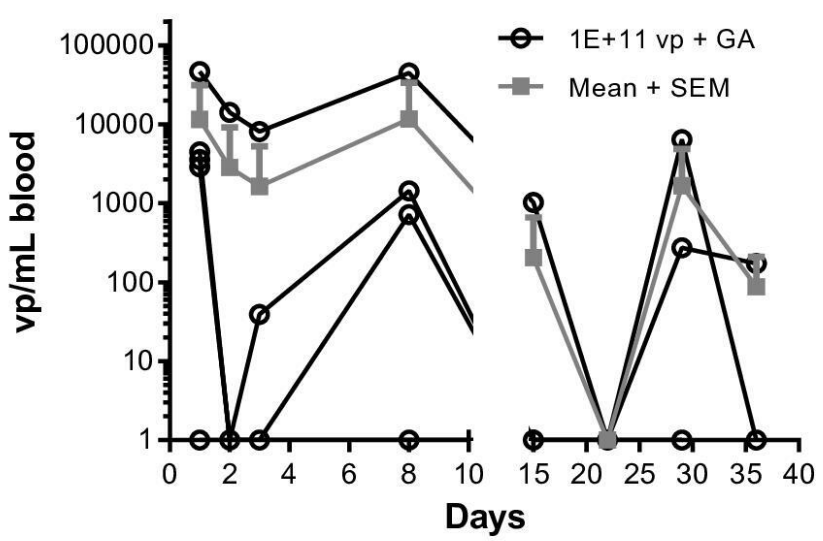

B)

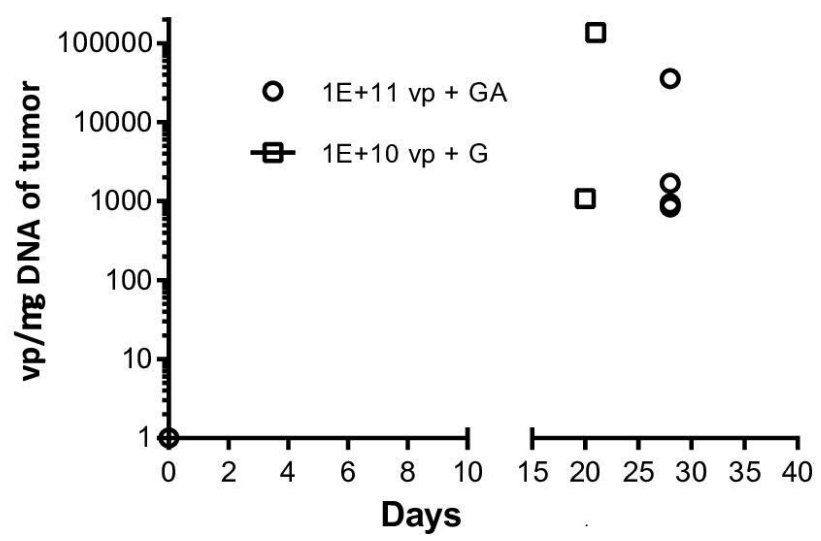

Figure 5 VCN-01 is detected in patient blood over time showing viral replication peaks and in tumors $20-28$ days after treatment. (A) Blood levels of VCN-01 at the indicated time points as determined by qPCR. Each black line represents a patient (six patients) and the mean of all patients treated with $1 \times 10^{11} \mathrm{vp}+$ nab-paclitaxel plus gemcitabine (GA) per time point is shown in gray. (B) VCN-01 is detected in all tumors analyzed on days 20-28. Fine-needle aspiration biopsy of the tumor performed at the indicated times (preVCN-01 or post-treatment) on each patient and analyzed by qPCR to detect the number of viral genomes (six patients). Each tumor sample is represented by a symbol, performed either on day 0 (negative) or between day 20 and 28 postVCN-01 it administration. G, gemcitabine.

was performed in four patients before and after treatment. As shown in figure $6 \mathrm{~B}$, treatment reduced tumor stiffness in the five assessed patients treated with VCN-01 combined with either gemcitabine or nab-paclitaxel plus gemcitabine. These results suggest that VCN-01 replicates within the tumor, leading to hyaluronidase expression with consequent reduction of tumor stiffness (even in the absence of nab-paclitaxel) that can potentially increase the extravasation of chemotherapy.

\section{DISCUSSION}

There is a significant need to develop new treatments for patients with PDAC. Oncolytic viruses are an attractive new class of cancer therapeutics. To date, two oncolytic
A)

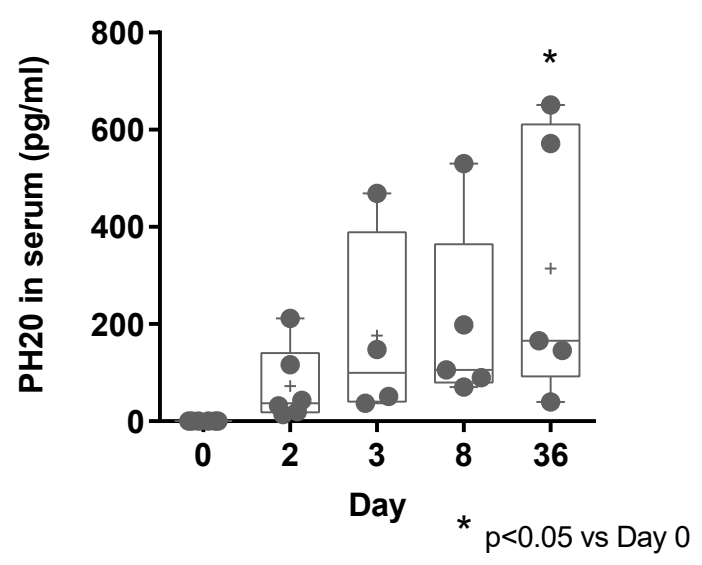

B)

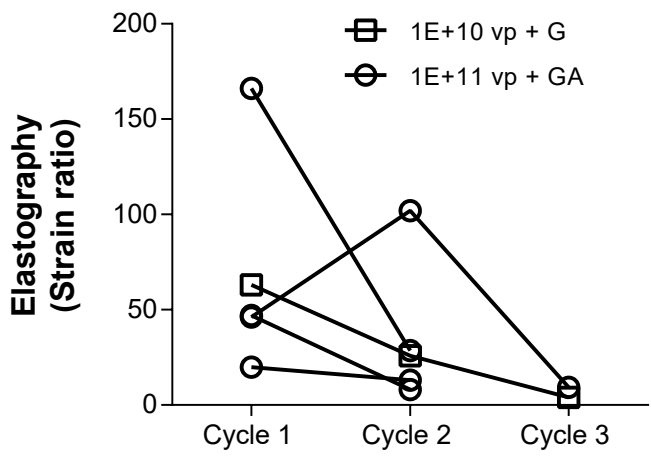

Figure 6 Hyaluronidase PH20 expression and stroma disruption effects of VCN-01 in patients. (A) PH2O serum levels as determined by ELISA at the indicated time points in patients treated with VCN-01 in combination with nabpaclitaxel plus gemcitabine (GA). Each filled circle represents a serum sample per patient. Analyzed patients were injected at a dose of $1 \times 10^{11} \mathrm{vp}$ of $\mathrm{VCN}-01$ (six patients). One-way ANOVA (Tukey's multiple comparisons test) was used to evaluate multiple comparisons between groups. $\mathrm{PH} 20$ expression in serum samples at different time points is expressed as $\mathrm{pg} / \mathrm{mL}$ minus background levels detected for each sample at day 0 and represented in box and whiskers graphs. (B) Changes in strain ratio measured by endoscopic ultrasound (EUS) elastography in five patients prior to cycles 1, 2 and 3. Each line represents an individual patient. ANOVA, analysis of variance.

genetically engineered viruses have been approved by regulatory agencies for clinical use as antitumor agents: Oncorine (H101) and IMLYGIC (talimogene laherparepvec, T-Vec). ${ }^{25}{ }^{26}$ VCN-01 is an oncolytic adenovirus engineered to express hyaluronidase. This agent is expected to have a dual mechanism of action including classic oncolysis as well as disruption of the HA-rich cancer stroma. The latter effect is expected to facilitate delivery and replication of the virus itself as well as the delivery of other agents. Furthermore, these two actions combined may lead to increased stromal inflammation ${ }^{27}$ 
and sensitization to immune-mediated treatments. Therefore, PDAC stands out as a candidate tumor type to explore the efficacy of VCN-01. These preclinical and clinical studies were designed to determine the efficacy, safety and mechanism of action of VCN-01 in PDAC.

We first conducted a series of preclinical studies to explore whether VCN-01 replicates in tumor tissues, disrupts HA, increases drug delivery, and exerts antitumor effects. Data presented here support the notion that VCN-01 administration resulted in these effects. Several important observations with implications for the subsequent clinical development of the agent were made. VCN-01 is effective both via intravenous and IT administration, leads to substantial and prolonged tumor control, and synergizes with chemotherapy. VCN-01 antitumor activity and replication potential observed after intravenous administration are important supporting the clinical development of this agent by this more facile route of administration. Furthermore, its limited replicative potential in normal liver also suggests that expected liver damage caused by this class of agents is likely to be transient. Accordingly, VCN-01 has also been administered intravenous in combination with chemotherapy in patients with advanced PDAC. ${ }^{27}$

A key differentiating factor of VCN-01 is its production of hyaluronidase PH20. Prior preclinical studies showed that HA elimination by pegylated hyaluronidase (PEGPH20) softens tumors, decompresses tumor vessels, increases chemotherapy delivery, and results in superior antitumor effects. ${ }^{152829}$ Here, we show that VCN-01 exerts similar effects including HA degradation, improved antitumor effects, and enhanced delivery of chemotherapy and therapeutic antibodies. We have previously shown that unarmed oncolytic adenoviruses do not degrade $\mathrm{HA},{ }^{19}$ but they increase fibrosis surrounding the infected areas. ${ }^{30}$ As nab-paclitaxel has been proposed as a stroma disrupting agent, ${ }^{23}$ it is important to highlight that VCN-01 increased the delivery of gemcitabine when administered alone. Of note, the better drug extravasation observed for VCN-01 occurs in spite of the surrounding fibrosis pointing to a complex interaction of the virus and the tumor stroma. In fact, oncolytic adenovirus therapy can be improved if cancer-associated fibroblasts are killed by the expression of a fibroblast activation protein-targeted bispecific T-cell engager. ${ }^{31}$ In addition, while some oncolytic viruses such as vaccinia and vesicular stomatitis virus can intrinsically induce vascular disruption, oncolytic adenoviruses do not and we and others have armed them with antiangiogenic factors to disrupt it. ${ }^{32}{ }^{33}$ Finally, it cannot be ruled out that the tumor debulking effect by the oncolysis might also have a minor impact in the drug extravasation effect. Although very recent positive advances have been made in PDAC treatment using BL-8040, pembrolizumab and chemotherapy, ${ }^{34}$ PDAC has been considered one of the tumor types for which check point inhibitors are not effective. ${ }^{35}{ }^{36}$ The increase in extravasation of PD-L1 antibody after VCN-01 treatment, together with the immune enhancing effect of IT VCN-01 in retinoblastoma, ${ }^{37}$ supports the development of this agent in combination with check point inhibitors in PDAC.

Two separate clinical trials evaluating systemic PEGPH20 (PEGylated PH20 with increased half-life) combined with nab-paclitaxel plus gemcitabine or FOLFIRINOX in patients with advanced PDAC were negative. ${ }^{38}{ }^{39}$ While the underlying reasons of this failure are not known, several hypotheses can be proposed. Toxicity was increased when chemotherapy was combined in both trials. This is not surprizing as PEGPH20 was given systemically and an increased delivery of chemotherapy to normal tissues may lead to higher toxicity. In one trial (HALO 301), because of the high rate of thrombotic events, patients were treated with prophylactic heparin that may have resulted in better outcome in the control arm. In addition, PEGPH20 was administered in a continued manner that may be deleterious as shown in some preclinical studies that disrupt tumor stroma for a long time (ie, blocking the sonic hedgehog pathway). ${ }^{40}$ Finally, it is not clear that the dose and schedule of administration of PEGPH20 were optimal in these studies.

Although these results may challenge the concept of targeting the PDAC stroma. VCN-01 uses a very different approach and combines the local expression of PH20 with intrinsic oncolytic effects and the induction of inflammation by both the viral infection as well as cell death. ${ }^{37}$ For these reasons, this agent showed enhanced effects in combination with immunotherapy agents and is currently being studied in a clinical trial where VCN-01 is combined with durvalumab (anti-PD-L1) (ClinicalTrials. gov Identifier: NCT03799744). Furthermore, because the viral effect is likely to be transient as the host immune system clears the virus, the stromal disruption effects are likely to be prominent shortly after administration and fade with time. It can be speculated that this dynamic may favor early drug delivery and immune system priming while preventing the angiogenesis and metastatic prone effects shown in studies in which the stroma was depleted chronically. ${ }^{40}$

Based on the encouraging preclinical data obtained with VCN-01, we conducted a proof-of-concept phase 1 trial of increasing doses of IT VCN-01 in patients with advanced PDAC. While in general the virus was well tolerated, one patient developed massive tumor necrosis, leading to a fatal hemorrhage on day 22 after treatment. This observation suggests a potent and rapid antitumor effect though the contribution of the concomitant chemotherapy or the injection procedure per se cannot be ruled out. Based on this serious adverse event together with the antitumor effects of VCN-01 in the tumor and the preclinical data supporting the feasibility of intravenous administration which is more convenient, we decided to concentrate further clinical development efforts in the intravenous administration route. ${ }^{27}$

Several lines of evidence suggest VCN-01 effectively replicated in tumor tissues. The pharmacokinetic profile of the virus in blood showed a biphasic peak which is consistent with an initial distribution phase, followed 
by later shedding or secondary viral replication peaks in blood as a result of intratumoral replication. This is further supported by the presence of viral particles in tumor cells as assessed in paired tumor biopsies collected before and after treatment. In addition, serum levels of PH20 increased after treatment administration, in agreement with viral replication and expression of the transgene. Consistent with these effects, injected tumor lesions remained stable. Increased viremia levels after injection may not be sufficiently high to cause effective VCN-01 delivery to non-injected tumors. These data emphasize the importance of treating patients with a high dose of VCN-01 systemically. ${ }^{27}$ In addition, there was a decrement in tumor stiffness in all VCN-01-injected lesions as measured by elastography, including a patient who did not receive nab-paclitaxel, an agent that has been shown to reduce tumor stiffness in prior clinical trials. ${ }^{23}$

In summary, the preclinical and clinical results presented here confirm the oncolytic capacity of VCN-01 in preclinical models and patients with PDAC. The agent effectively replicated in tumor tissues and expressed hyaluronidase, which in turn disrupted tumor stroma and facilitated drug delivery. These results support the evaluation of the efficacy of VCN-01 in combination with chemotherapy and/or immunotherapies for the treatment of advanced PDAC. Given the mechanism of action of VCN01 , the combination of VCN-01 with other treatments in cancers that have high HA content is also warranted.

\section{Author affiliations}

${ }^{1}$ VCN Biosciences, Sant Cugat del Valles, Barcelona, 08174, Spain

${ }^{2}$ Oncology Department, Hospital Universitario 12 de Octubre, Imas12, UCM, CNIO, CIBERONC, Madrid, Spain

${ }^{3}$ Medical Oncology Department, IDIBELL-Institut Catala d' Oncologia, L'Hospitalet de Llobregat, Barcelona, 08908, Spain

${ }^{4}$ Centro Integral Oncológico Clara Campal (CIOCC), Oña 10, 28050, Madrid, Spain

${ }^{5}$ Virotherapy and Gene Therapy Group, Oncobell and ProCure Programs, IDIBELLInstituto Catalan d'Oncología, L'Hospitalet de Llobregat, Barcelona, Spain

${ }^{6}$ Department of Hematology and Oncology, Institut d'Investigacions Biomèdiques August Pi i Sunyer (IDIBAPS), Hospital Clinic, Barcelona, Spain

${ }^{7}$ Hereditary Cancer Program, Oncobell Program, CIBERONC, IDIBELL-Instituto

Catalan d'Oncología, I'Hospitalet de Llobregat, Barcelona, Spain

${ }^{8}$ Endoscopic Unit, Servicio Aparato Digestivo, University Hospital 12 De 0ctubre, Madrid, Spain

${ }^{9}$ Hospital Universitari de Bellvitge, IDIBELL, Universitat de Barcelona, L'Hospitalet de Llobregat, Barcelona, Spain

${ }^{10}$ Div. of Hematology and Medical Oncology, Weill Cornell Medicine, New York, New York, USA

\section{Twitter Ramon Salazar @RamonSalazarS}

Acknowledgements The study team thanks patients and families for their willingness to participate in the clinical trial of VCN-01 by intratumor administration. Thanks to Marti Farrera and Silvia Torres-Manjon for technical assistance and processing biological samples. Graphical Abstract was created with BioRender.com.

Contributors Conception and design: MB-P, RAle, MC and MH. Development of methodology: AM-B, MG-A, SM, AR-G, RM, MMG and MVM. Acquisition of data (provided animals, acquired and managed patients, provided facilities, etc): RG-C, BL, RAlv, MCR, MP-C, JBG, SP, RS and MH. Analysis and interpretation of data (eg, statistical analysis, biostatistics, computational analysis): MB-P, AM-B, MG-A, SM, MVM, SP, RAle, EB and MC analyzed and interpreted non-clinical data; MB-P, RGS, $\mathrm{GC}, \mathrm{RAlv}, \mathrm{EB}, \mathrm{CB}, \mathrm{MC}$ and $\mathrm{MH}$ analyzed and interpreted clinical data. Writing, review and/or revision of the manuscript: MB-P, SP and MH wrote the manuscript; all authors revised and approved the manuscript. Administrative, technical, or material support (ie, reporting or organizing data, constructing databases): $M B-P, C B, R G-C$, RAlv, RS, MC and MH. Study Supervision: MB-P, CB, RG-C, RAlv, RS, MC and MH.

Funding MB-P, MG-A, SM, EB and MC were funded by CDTI (PANCATHER project IDI-20130759). The clinical study was supported by VCN Biosciences.

Competing interests MB-P, AM-B, MG-A, SM, MVM, EB, CB and MC are employees and RAIm and MH are consultants for VCN Biosciences. MC and RAlm are co-inventors of one patent application concerning the expression of hyaluronidase by oncolytic adenoviruses. MC, RAlm and GC have ownership interest in VCN Biosciences. MH is a founder and stock holder of Champions Oncology and Nelum Pharmaceuticals; he is a stock holder of Agenus, Pharmacyte, Highlight Pharmaceuticals, Oncomatrix, Inxmed and BMS; he is Board of Directors at BMS; he has received research support from Erytech, PanCan, TBA alliance and has received honorarium from Agenus, Oncomatrix, InxMed, Khar, Genechem, Cantargia, BMS, and royalties from Myriad and Kahr. RGC has provided scientific advice and/or received honoraria or funding for continuous medical education from AAA, Advanz Pharma, Amgen, Bayer, BMS, HMP, Ipsen, Merck, Mida-tech Pharma, MSD, Novartis, PharmaMar, Pfizer, Pierre Fabre, Roche, Servier and Sanofi, and has received research support from Pfizer, BMS and MSD.

\section{Patient consent for publication Not applicable.}

Ethics approval All animal studies were performed at the IDIBELL facility (AAALAC unit 1155) and approved by the IDIBELL's Ethical Committee for Animal Experimentation. The clinical study was conducted at three hospitals in Spain and was approved by the pertinent health authorities and ethics committees and adhered to the ethical principles of the Declaration of Helsinki and was performed in compliance with Good Clinical Practice.

Provenance and peer review Not commissioned; externally peer reviewed.

Data availability statement All data relevant to the study are included in the article or uploaded as online supplemental information. AcknowledgmentsThe study team thanks patients and families for their willingness to participate in the clinical trial of VCN-01 by intratumor administration. Thanks to Marti Farrera and Silvia Torres-Manjon for technical assistance and processing biological samples. Graphical Abstract was created with BioRender.com.

Supplemental material This content has been supplied by the author(s). It has not been vetted by BMJ Publishing Group Limited (BMJ) and may not have been peer-reviewed. Any opinions or recommendations discussed are solely those of the author(s) and are not endorsed by BMJ. BMJ disclaims all liability and responsibility arising from any reliance placed on the content. Where the content includes any translated material, BMJ does not warrant the accuracy and reliability of the translations (including but not limited to local regulations, clinical guidelines, terminology, drug names and drug dosages), and is not responsible for any error and/or omissions arising from translation and adaptation or otherwise.

Open access This is an open access article distributed in accordance with the Creative Commons Attribution Non Commercial (CC BY-NC 4.0) license, which permits others to distribute, remix, adapt, build upon this work non-commercially, and license their derivative works on different terms, provided the original work is properly cited, appropriate credit is given, any changes made indicated, and the use is non-commercial. See http://creativecommons.org/licenses/by-nc/4.0/.

\section{ORCID iDs}

Miriam Bazan-Peregrino http://orcid.org/0000-0001-7367-8304

Manuel Hidalgo http://orcid.org/0000-0002-3765-3318

\section{REFERENCES}

1 Korc M. Pancreatic cancer-associated stroma production. Am J Surg 2007;194:S84-6.

2 Sun C, Ansari D, Andersson R, et al. Does gemcitabine-based combination therapy improve the prognosis of unresectable pancreatic cancer? World J Gastroenterol 2012;18:4944-58.

3 Rasheed ZA, Matsui W, Maitra A. Pathology of pancreatic stroma in PDAC. In: Grippo PJ, Munshi HG, eds. Pancreatic cancer and tumor microenvironment. Trivandrum (India), 2012.

4 Nielsen MFB, Mortensen MB, Detlefsen S. Key players in pancreatic cancer-stroma interaction: cancer-associated fibroblasts, endothelial and inflammatory cells. World J Gastroenterol 2016;22:2678-700.

5 Chu GC, Kimmelman AC, Hezel AF, et al. Stromal biology of pancreatic cancer. J Cell Biochem 2007;101:887-907.

6 Erkan M, Reiser-Erkan C, Michalski CW, et al. Tumor microenvironment and progression of pancreatic cancer. Exp Oncol 2010;32:128-31. 
7 Whatcott CJ, Han H, Posner RG, et al. Targeting the tumor microenvironment in cancer: why hyaluronidase deserves a second look. Cancer Discov 2011;1:291-6.

8 Toole BP. Hyaluronan: from extracellular glue to pericellular cue. Nat Rev Cancer 2004;4:528-39.

9 Bertrand P, Girard N, Delpech B, et al. Hyaluronan (hyaluronic acid) and hyaluronectin in the extracellular matrix of human breast carcinomas: comparison between invasive and non-invasive areas. Int J Cancer 1992;52:1-6.

10 Seufferlein T, Ducreux M, Hidalgo M, et al. More than a Gel \& Hyaluronic Acid, a Central Component in the Microenvironment of Pancreatic Cancer. Eur Oncol Haematol 2018;14:40-4.

11 Oettle H, Post S, Neuhaus P, et al. Adjuvant chemotherapy with gemcitabine vs observation in patients undergoing curative-intent resection of pancreatic cancer: a randomized controlled trial. JAMA 2007;297:267-77.

12 Minchinton AI, Tannock IF. Drug penetration in solid tumours. Nat Rev Cancer 2006;6:583-92.

13 Feig C, Gopinathan A, Neesse A, et al. The pancreas cancer microenvironment. Clin Cancer Res 2012;18:4266-76.

14 Nakazawa $\mathrm{H}$, Yoshihara S, Kudo D, et al. 4-Methylumbelliferone, a hyaluronan synthase suppressor, enhances the anticancer activity of gemcitabine in human pancreatic cancer cells. Cancer Chemother Pharmacol 2006;57:165-70.

15 Provenzano PP, Cuevas C, Chang AE, et al. Enzymatic targeting of the stroma ablates physical barriers to treatment of pancreatic ductal adenocarcinoma. Cancer Cell 2012;21:418-29.

16 Singha NC, Nekoroski T, Zhao C, et al. Tumor-Associated hyaluronan limits efficacy of monoclonal antibody therapy. Mol Cancer Ther 2015;14:523-32.

17 Hendifar A, Bullock A. Breaking the Barrier-PEGylated RecombinantHuman Hyaluronidase (PEGPH20) - A New Therapeutic Approach to the Treatment of Pancreatic Ductal Adenocarcinoma. Oncol Hematol Rev 2017:13:107-11.

18 Rodríguez-García A, Giménez-Alejandre M, Rojas JJ, et al. Safety and efficacy of VCN-01, an oncolytic adenovirus combining fiber HSG-binding domain replacement with RGD and hyaluronidase expression. Clin Cancer Res 2015;21:1406-18.

19 Guedan S, Rojas JJ, Gros A, et al. Hyaluronidase expression by an oncolytic adenovirus enhances its intratumoral spread and suppresses tumor growth. Mol Ther 2010;18:1275-83.

20 Vives M, Ginestà MM, Gracova K, et al. Metronomic chemotherapy following the maximum tolerated dose is an effective anti-tumour therapy affecting angiogenesis, tumour dissemination and cancer stem cells. Int J Cancer 2013;133:2464-72.

21 Janssen J, Schlörer E, Greiner L. EUS elastography of the pancreas: feasibility and pattern description of the normal pancreas, chronic pancreatitis, and focal pancreatic lesions. Gastrointest Endosc 2007;65:971-8.

22 Iglesias-Garcia J, Lindkvist B, Lariño-Noia J, et al. Endoscopic ultrasound elastography. Endosc Ultrasound 2012;1:8-16.

23 Alvarez R, Musteanu M, Garcia-Garcia E, et al. Stromal disrupting effects of nab-paclitaxel in pancreatic cancer. $\mathrm{Br} J$ Cancer 2013;109:926-33.

24 Hecht JR, Bedford R, Abbruzzese JL, et al. A phase I/II trial of intratumoral endoscopic ultrasound injection of ONYX-015 with intravenous gemcitabine in unresectable pancreatic carcinoma. Clin Cancer Res 2003:9:555-61.
25 Liang M, Oncorine LM. Oncorine, the world first oncolytic virus medicine and its update in China. Curr Cancer Drug Targets 2018;18:171-6.

26 Conry RM, Westbrook B, McKee S, et al. Talimogene laherparepvec: first in class oncolytic virotherapy. Hum Vaccin Immunother 2018;14:839-46.

27 Garcia-Carbonero R, Gil Martín M, Alvarez Gallego R, et al. Systemic administration of the hyaluronidase-expressing oncolytic adenovirus VCN-01 in patients with advanced or metastatic pancreatic cancer: first-in-human clinical trial. Annals Oncol 2019;30:v271-2.

28 Thompson CB, Shepard HM, O'Connor PM, et al. Enzymatic depletion of tumor hyaluronan induces antitumor responses in preclinical animal models. Mol Cancer Ther 2010;9:3052-64.

29 Jacobetz MA, Chan DS, Neesse A, et al. Hyaluronan impairs vascular function and drug delivery in a mouse model of pancreatic cancer. Gut 2013;62:112-20.

30 Cascallo M, Alonso MM, Rojas JJ, et al. Systemic toxicity-efficacy profile of ICOVIR-5, a potent and selective oncolytic adenovirus based on the pRB pathway. Mol Ther 2007;15:1607-15.

31 de Sostoa J, Fajardo CA, Moreno R, et al. Targeting the tumor stroma with an oncolytic adenovirus secreting a fibroblast activation proteintargeted bispecific T-cell engager. J Immunother Cancer 2019;7:19.

32 Toro Bejarano M, Merchan JR. Targeting tumor vasculature through oncolytic virotherapy: recent advances. Oncolytic Virother 2015;4:169-81.

33 Bazan-Peregrino M, Sainson RCA, Carlisle RC, et al. Combining virotherapy and angiotherapy for the treatment of breast cancer. Cancer Gene Ther 2013;20:461-8.

34 Bockorny B, Semenisty V, Macarulla T, et al. BL-8040, a CXCR4 antagonist, in combination with pembrolizumab and chemotherapy for pancreatic cancer: the combat trial. Nat Med 2020;26:878-85.

35 Royal RE, Levy C, Turner K, et al. Phase 2 trial of single agent ipilimumab (anti-CTLA-4) for locally advanced or metastatic pancreatic adenocarcinoma. J Immunother 2010;33:828-33.

36 O'Reilly EM, DY O, Dhani N. Durvalumab with or without tremelimumab for patients with metastatic pancreatic ductal adenocarcinoma: a phase 2 randomized clinical trial. JAMA Oncol 2019.

37 Pascual-Pasto G, Bazan-Peregrino M, Olaciregui NG, et al. Therapeutic targeting of the RB1 pathway in retinoblastoma with the oncolytic adenovirus VCN-01. Sci Transl Med 2019;11. doi:10.1126/scitransImed.aat9321. [Epub ahead of print: 2301 2019].

38 Ramanathan RK, McDonough SL, Philip PA, et al. Phase lb/ II randomized study of Folfirinox plus PEGylated recombinant human hyaluronidase versus Folfirinox alone in patients with metastatic pancreatic adenocarcinoma: SWOG S1313. J Clin Oncol 2019;37:1062-9.

39 Tempero MA, Van Cutsem E, Sigal D, et al. HALO 109-301: A randomized, double-blind, placebo-controlled, phase 3 study of pegvorhyaluronidase alfa (PEGPH20) + nab-paclitaxel/gemcitabine (AG) in patients (pts) with previously untreated hyaluronan (HA)high metastatic pancreatic ductal adenocarcinoma (mPDA). JCO 2020;38:638-38.

40 Rhim AD, Oberstein PE, Thomas DH, Rhim Andrew D, Thomas Dafydd $\mathrm{H}$, et al. Stromal elements act to restrain, rather than support, pancreatic ductal adenocarcinoma. Cancer Cell 2014;25:735-47. 